\title{
Association of BDNFVal66Met polymorphism with HPA and SAM axis reactivity to psychological and physical stress
}

This article was published in the following Dove Press journal:

Neuropsychiatric Disease and Treatment

II November 2014

Number of times this article has been viewed

\author{
Jusen Tsuru' \\ Yoshihiro Tanaka' \\ Yoshinobu Ishitobi' \\ Yoshihiro Maruyama' \\ Ayako Inoue' \\ Aimi Kawano' \\ Rie Ikeda' \\ Tomoko Ando' \\ Harumi Oshita ${ }^{2}$ \\ Saeko Aizawa ${ }^{1}$ \\ Koji Masuda' \\ Haruka Higuma' \\ Masayuki Kanehisa' \\ Taiga Ninomiya' \\ Jotaro Akiyoshi'
}

'Department of Neuropsychiatry, ${ }^{2}$ Department of Applied Linguistics, Faculty of Medicine, Oita University, Oita, Japan
Correspondence: Jotaro Akiyoshi Department of Neuropsychiatry, Faculty of Medicine, Oita University, Hasama-Machi, Oita 879-5593, Japan

Tel +8I 975865823

Fax +8I 975493583

Email akiyoshi@oita-u.ac.jp
Background: Decreased expression of brain-derived neurotrophic factor (BDNF) is implicated in enhanced stress responses. The BDNF Val66Met polymorphism is associated with psychological changes; for example, carriers of the Met allele exhibit increased harm avoidance as well as a higher prevalence of depression and anxiety disorder.

Methods: To analyze the effects of BDNF Val66Met on stress responses, we tested 226 university students ( 88 women and 138 men) using a social stress procedure (Trier Social Stress Test [TSST]) and an electrical stimulation stress test. Stress indices were derived from repeated measurements of salivary $\alpha$-amylase, salivary cortisol, heart rate, and psychological testing during the stress tests. All subjects were genotyped for the Val66Met polymorphism (G196A).

Results: A significant three-way interaction (time [3 levels] $\times$ BDNF [Val/Val, Val/Met, Met/ Met]; $P<0.05$ ) was demonstrated that revealed different salivary cortisol responses in the TSST but not in electrical stimulation. Met/Met women had stronger cortisol responses than Val/Met and $\mathrm{Val} / \mathrm{Val}$ individuals in the TSST. Met/Met men exhibited stronger salivary cortisol responses than $\mathrm{Val} / \mathrm{Met}$ and $\mathrm{Val} / \mathrm{Val}$ individuals in the TSST.

Conclusion: These results indicate that a common, functionally significant polymorphism in BDNF had different effects on hypothalamic-pituitary-adrenocortical axis reactivity but not on sympathetic adrenomedullary reactivity in TSST and electrical stimulation tests.

Keywords: stress, brain-derived neurotrophic factor, cortisol, saliva

\section{Introduction}

Research on people's reaction to high levels of stress has established that a neuroendocrine marker plays an important role in this reaction. ${ }^{1-3}$ The stress reaction is mainly regulated by an axial system consisting of two neuroendocrine systems: the hypothalamic-pituitary-adrenocortical (HPA) and sympathetic adrenomedullary (SAM) systems. The role of the HPA axis activity in mediation of the stress response has been investigated for decades. The HPA axis is a part of the complicated neuroendocrine mechanism involved in the regulation of bioactivity in response to a stimulus, change in environment, or confrontation, and thus forms an important aspect of stress responses. Cortisol is an essential hormone controlling the rule of a stress reaction. Although serum cortisol is either in a free (active) or protein-bound (inactive) form, salivary cortisol is found exclusively in the free form. The level of circulating free cortisol in the saliva is used as an index of noninvasiveness and HPA axis activity. ${ }^{4}$ Salivary cortisol levels increase several times rapidly in the event of psychological stress, ${ }^{5}$ as observed in events of physical stress such as firefighting ${ }^{6}$ or cold pressor stress. ${ }^{7}$ A possible association between stress and the salivary $\alpha$-amylase (sAA) level 
has recently received considerable attention; however, rigorous psychosocial studies of $\alpha$-amylase responsiveness are difficult because of the complexity of the system.

Secretion of salivary $\alpha$-amylase is controlled by the beta adrenergic receptor blockade, ${ }^{8}$ and its levels are used as a biomarker of psychosocial stress responses of the SAM system. ${ }^{9,10}$ sAA levels increase markedly in response to high-stress tasks or procedures, such as parachute jumps, ${ }^{11}$ a stressful video game, ${ }^{12}$ or psychological stress observed in students, such as that which occurs before an academic test. ${ }^{13,14}$ Past research has demonstrated the secretion of sAA as a response to a psychosocial stress, using the Trier Social Stress Test (TSST), ${ }^{15-17}$ and sAA secretion is also seen in individuals who underwent functional magnetic resonance imaging examination after viewing pictures associated with negative feelings, ${ }^{7}$ and in individuals who were subjected to dynamic video stress. ${ }^{18}$ Pharmacologic manipulation of the SAM system underscores the reliability of sAA as a measure of sympathetic activity. Stimulation of the SAM system by the $\alpha_{2}$-adrenergic receptor antagonist yohimbine significantly increases the levels of sAA. ${ }^{19}$

Activation of the stress response by an experience that is not fright, denial, or expectation activates a reaction from the nervous system in addition to that from the continuous neuroendocrine system. The SAM system operates through catecholamine (noradrenaline and adrenaline) signals and interacts with glucocorticoids from the HPA system; the sympathetic nervous system has an important role in homeostasis and stress responses. ${ }^{20}$ The various roles of glucocorticoids in stress reactivity and their interactions with the associated neuroendocrine systems have been investigated widely. ${ }^{21}$ Glucocorticoid activity stimulates and deactivates stress responses. A study of the interactions of the SAM and glucocorticoid-HPA systems would help elucidate the mechanism of stress responses. ${ }^{7}$ Some studies suggest associations between responsiveness of the HPA axis to TSST and the glucocorticoid receptor gene, ${ }^{22}$ the GABRA6 gene, ${ }^{23}$ and the $\mu$-opioid receptor gene. ${ }^{24}$ Although some researchers have investigated stress responses to pain, ${ }^{25-29}$ most studies have been conducted in patients with psychiatric disorders including anxiety disorder, trauma, and chronic sharp pain. It is not clear how the stressor associated with pain interacts with these two stress response systems. Furthermore, very few studies have compared the SAM and HPA systems in the reaction to psychological and physical stress. ${ }^{7}$ Recently, the temporal pattern of sAA and a salivary cortisol stress response has been reported. ${ }^{20}$ In 2012 , Campbell and Ehlert reported that differences between acute physiological and emotional stress cannot be estimated by means of pathological markers; however, these differences could represent a normal reactivity pattern and/or be a result of various methodological issues. ${ }^{30}$

Our research pertains to the role of brain-derived neurotrophic factor (BDNF) in the regulation of the responses of the HPA axis to social stress. BDNF, a member of the neurotrophin growth factor family, ${ }^{31}$ has a pervasive influence on central nervous system development, and later in life, it is involved in neural survival and differentiation, and in the plasticity of the central nervous system. ${ }^{32}$ Occurrence of stress early in life alters the threshold of HPA reactivity in humans and other animals; therefore, genes related to physiological plasticity and to the protective effects of BDNF against stressinduced neuronal damage are of considerable interest. ${ }^{33}$

BDNF, similar to other peptide growth factor genes, encodes a precursor peptide (proBDNF) that is proteolytically cleaved to produce the mature protein. ${ }^{34}$ A single, frequent, nonconservative polymorphism in the human BDNF gene (dbSNP rs6265) is a known single-nucleotide polymorphism (SNP) at nucleotide 196 (G/A), resulting in a valine-tomethionine substitution at codon 66 (Val66Met). Several studies have demonstrated the functional influence of the Val66Met polymorphism on the BDNF expression level, using brain imaging and transfection. ${ }^{35,36}$ Furthermore, in human and animal models, Val66Met is associated with stress-related aberrations, such as anxiety-related personality traits $^{37}$ and depressive disorder. ${ }^{10,38}$ In addition, BDNF Met allele carriers might be at risk of developing smaller hippocampal volumes and might be susceptible to major depressive disorder. ${ }^{39}$ Patients with clinical depression carrying the Met allele (BDNF Val/Met and BDNF Met/Met) are more likely to suffer from suicidal ideation and are more likely to attempt suicide. ${ }^{40}$ With respect to exposure to high levels of stress early in life, carriers of the Met allele were found to have smaller hippocampal volumes and syndromal depression, whereas Val/Val homozygotes showed no effect on depression. ${ }^{41}$ BDNF Val/Met and BDNF Met/Met individuals also respond better to antidepressant treatment compared with BDNF Val/Val homozygotes. ${ }^{42,43}$

In line with its roles in maintaining neuronal integrity and plasticity, BDNF may play a role in the etiology of stress-related traits and disorders, such as depression and anxiety. ${ }^{10,44}$ Because of the prevalence of the Val66Met polymorphism and its effect on the levels of the BDNF protein, we are interested in its potential role in modulating salivary cortisol and autonomic response in nonclinical subjects during TSST and clinical stimulation stress tests. 
The target of this research was to investigate the response of the two main neuroendocrine systems (HPA and SAM axes) to two stressors in individuals with different BDNF genotypes. To evaluate this response, we measured the sAA levels and salivary cortisol responses to a psychosocial stress task (TSST) and to physical stress (electrical stimulation) in healthy volunteers. Stress reactivity differs between men and women. Some studies in men and women have suggested that the reactions to stress differ in individual evaluation and memory inspection regarding an affective matter. ${ }^{45-48}$ van Stegeren et $\mathrm{al}^{48}$ have shown that men have a stronger saliva cortisol response than women after exposure to stressors. ${ }^{49}$ However, only a few studies have demonstrated a difference in sAA levels and the cortisol response between men and women. ${ }^{17,50,51}$ Therefore, we investigated the influences of sex on sAA, as well as on cortisol responses. We tested 226 university students using the TSST and a physical stress procedure. Stress indices were derived from repeated measurements of sAA levels, salivary cortisol, heart rate, and psychological testing during TSST and electrical stimulation tests; all subjects were genotyped for the Val66Met polymorphism (G196A in BDNF).

\section{Materials and methods Subjects}

The study population consisted of healthy volunteers $(n=235)$ with no history of mental illness. None of the participants took any medication during the tests. Exclusion criteria included the use of tobacco, pregnancy, steroid medication within the last 3 years, body mass index of $32 \mathrm{~kg} / \mathrm{m}^{2}$ or higher, mental disorders, and physical diseases. All participants had Beck Depression Inventory scores of 7 or lower and had no history of depression. We excluded medical illness and other psychiatric disorders using the Mini International Neuropsychiatric Interview. Three individuals did not respond to the questionnaire (two men and one woman), one individual did not match the selection criteria (one woman; social anxiety disorder), 20 individuals refused to participate in the TSST and/or electric stimulation tests (14 men and six women), one woman withdrew because of acute abdominal pain during rest periods, and eleven individuals were excluded for technical reasons. The final study population included 226 people ( 88 women and 138 men; average age, 24.9 years; range, 22-45 years). All remaining participants underwent both tests (the electrical stimulation stress test and the TSST [a 5-minute speech task plus a 5-minute mental arithmetic task]) at random. Participants included five physicians, seven clerks, and 214 students of Oita University in Japan. Informed written consent was obtained from all participants. The protocol of this study was approved by the ethics committee of Oita University.

\section{Procedures}

All participants underwent both electrical stimulation and TSST stress tests over the period of 1 week. To eliminate the possibility of them becoming relaxed or accustomed to the experimental environment, the participants were divided into small groups, each consisting of four or five individuals. Each group underwent electrical stimulation or TSST alternately in the first experimental test. The second experimental test was carried out 1 week after the first. The participants were instructed to refrain from taking any medication 5 hours before arrival and to refrain from brushing their teeth or eating at least 60 minutes before the tests. We measured saliva cortisol levels and sAA three times (immediately before, immediately after, and 20 minutes after the intervention) in a manner consistent with previous reports. ${ }^{52,53}$ In addition, we examined the interaction of salivary cortisol levels and sAA levels. To minimize the effects of circadian variation in salivary cortisol and sAA, collection of saliva and exposure to psychological and physical stress factors were conducted between $1 \mathrm{pm}$ and $4 \mathrm{pm} .{ }^{54}$ To exclude the influence of thermal stress, experiments were performed at a constant temperature of $25^{\circ} \mathrm{C} \pm 2^{\circ} \mathrm{C}$.

During the experiment, the participants wore stimulator coils connected to a stimulator on the wrist. ${ }^{26}$ This device passed a current through the motor and sensory fibers of the median nerve in the right wrist. Electric stimulation of threshold stimulus level, defined as the strongest stimulation the participants could withstand, was given for 40 seconds to each subject. The strength of the electrical stimulation was between 2 and $55 \mathrm{~mA}$, with its intensity varying between participants. The participants were told that the electrical stimulation would cause pain but that it would not cause damage or burn the skin. Electrical stimulation of short duration reportedly causes no physical damage. ${ }^{26}$ The differences in the strength of electrical stimulation in different subjects may depend on the individual differences in psychological factors related to depression and anxiety disorders. ${ }^{4,51}$ The intensity of the stimulation was determined for each participant, as described earlier. Therefore, the setting of our experiments, using this level of pain, is consistent with ethical standards for studies with humans.

All participants entered the laboratory 30 minutes before the experiment to minimize physical activity, prior stress, and emotional variation. They filled fill out a questionnaire, and some participants received a stress test. ${ }^{4}$ The TSST was composed of a 3-minute preparation period, a 5-minute speech task 
(patients were asked to describe their personal characteristics), and a 5-minute mental arithmetic task in front of interviewers. After the stress task, participants stayed in the laboratory for 20 minutes for sample collection during recovery.

\section{Sampling and biochemical analysis}

The sAA levels were measured using a dry chemistry system (Nipro Inc., Osaka, Japan) according to the manufacturer's protocol. This method, using a handheld monitor, enables measurements to be made easily and quickly. ${ }^{55}$ The handheld device is composed of a monitor and disposable test strip. ${ }^{50,51}$ This method of analyzing the sAA level has been evaluated objectively. ${ }^{56,57}$ Saliva was sampled by placing the test strip under the tongue for 30 seconds. ${ }^{58}$ Thereafter, the strip was immediately placed in a saliva automatic transfer system and the saliva was moved to the $\alpha$-amylase test paper on the reverse side of the strip by means of a compression sleeve. The $\alpha$-amylase test paper consists of a substrate containing 2-chloro-4-nitrophenyl-4-Ob-D-galactopyranosylmaltoside, which leads to an enzymatic reaction. The free 2-chloro-4-nitrophenol levels were measured optically after 20 seconds. One unit of $\alpha$-amylase activity was defined as that which reduced a sugar equivalent to $1 \mathrm{mmol} /$ minute of maltose. The concentration of salivary cortisol was analyzed using an enzyme-linked immunosorbent assay, which had intraassay and interassay coefficients of $3 \%$ and $10 \%$, respectively. We stored samples at $-20^{\circ} \mathrm{C}$ until analysis.

We administered the State and Trait versions of the Spielberger Anxiety Inventory (STAI-S and STAI-T, respectively) ${ }^{59}$ to participants before performing the electrical stimulation and TSST protocols. We also administered the Profile of Mood States (POMS); ${ }^{55}$ the Neuroticism, Extraversion, and Openness (NEO) Personality Inventory Revised (NEO-PI-R); ${ }^{60}$ and the Temperament and Character Inventory (TCI) tests to participants before performing the electrical stimulation and TSST protocols. ${ }^{61}$ Finally, we also examined the high-frequency (HF) and low-frequency (LF) heart rate variability, and LF/HF ratio, immediately after electrical stimulation and TSST by means of an APG Heart-Rater SA 3000P (Tokyo Iken Co., Ltd., Japan).

\section{DNA extraction and genotyping}

DNA was extracted from blood samples using a DNA Extractor SP Kit (WAKO, Osaka, Japan). SNP (rs6265, A/G; $\mathrm{Val} / \mathrm{Met}$ ) was measured using $\mathrm{TaqMan}^{\circledR}$ polymerase chain reaction (PCR) SNP genotyping assays on a Roche LightCycler480 (Basel, Switzerland). Ten microliter PCR reactions were performed with 2 ng genomic DNA, using TaqMan ${ }^{\circledR}$ single nucleotide polymorphism genotyping assays (Applied Biosystems, Foster City, CA, USA) including the Universal PCR master mix, according to standard protocols.

\section{Statistical analysis}

We separated all analyses by sex and adjusted for age. The data were presented as mean \pm standard deviation. We analyzed the data using the SPSS statistical package, version 19 (SPSS Inc., IL, USA). The $\chi^{2}$ test and $t$-test were used for the descriptive characteristics. A two-way analysis of variance, followed by the Bonferroni post hoc comparison test, was used to compare sAA and cortisol responses. $P$-values $<0.05$ were considered statistically significant.

\section{Results \\ Sociodemographics and clinical characteristics of the three BDNF genotypes}

The sociodemographics and clinical characteristics of the three BDNF genotypes are shown in Table 1. The three groups did not differ by sex, age, or current smoker status.

\section{Self-reported measures of stress}

There were no significant differences among the $\mathrm{Val} / \mathrm{Val}$, $\mathrm{Val} / \mathrm{Met}$, and Met/Met groups for the following variables:

Table I Sociodemographic data, BDI, and STAI scores for the three BDNF genotype groups

\begin{tabular}{|c|c|c|c|c|c|}
\hline Characteristic & $\mathrm{Val} / \mathrm{Val}(\mathrm{n}=46)$ & Val/Met $(n=85)$ & Met/Met (n=97) & F or $\chi^{2}$ & $\mathbf{P}$ \\
\hline Age, years \pm standard deviation & $24.7 \pm 2.8$ & $25.5 \pm 4.9$ & $24.9 \pm 3.5$ & I & 0.37 \\
\hline Sex, male/female & $21 / 25$ & $56 / 29$ & $51 / 46$ & 5.83 & 0.054 \\
\hline Current smoker, $\mathrm{n}$ & 3 & 5 & I & 3.82 & 0.148 \\
\hline Beck Depression Inventory (BDI) & $3.27 \pm 5.01$ & $3.62 \pm 5.35$ & $3.31 \pm 4.16$ & 0.11 & 0.89 \\
\hline \multicolumn{6}{|l|}{ STAI (TSST) } \\
\hline Trait & $48.33 \pm 11.10$ & $47.46 \pm 8.74$ & $47.25 \pm 9.01$ & 1.23 & 0.33 \\
\hline State & $49.98 \pm 10.85$ & $48.62 \pm 9.36$ & $48.30 \pm 8.82$ & 1.03 & 0.36 \\
\hline \multicolumn{6}{|l|}{ STAI (electrical stimulation) } \\
\hline Trait & $48.33 \pm I 1.22$ & $47.45 \pm 8.74$ & $47.06 \pm 9.01$ & 1.79 & 0.17 \\
\hline State & $50.18 \pm 10.89$ & $48.79 \pm 9.39$ & $48.14 \pm 8.83$ & 1.80 & 0.17 \\
\hline
\end{tabular}

Notes: Data represent mean \pm standard deviation unless otherwise noted. $F$ ratios from one-way analyses of variance followed by Bonferroni post hoc comparisons. Abbreviations: BDNF, brain-derived neurotrophic factor; STAI, Spielberger Anxiety Inventory; TSST, Trier Social Stress Test. 
Table 2 POMS scores for the three BDNF genotype groups

\begin{tabular}{|c|c|c|c|c|c|}
\hline & $\mathrm{Val} / \mathrm{Val}(\mathrm{n}=46)$ & Val/Met $(n=85)$ & Met/Met $(n=97)$ & $\boldsymbol{F}$ & $P$ \\
\hline \multicolumn{6}{|c|}{ POMS (TSST) } \\
\hline T-A & $48.33 \pm 11.10$ & $47.46 \pm 8.74$ & $47.25 \pm 9.01$ & & \\
\hline $\mathrm{D}$ & $49.98 \pm 10.85$ & $48.62 \pm 9.36$ & $48.30 \pm 8.82$ & & \\
\hline \multirow[t]{2}{*}{$\mathrm{A}-\mathrm{H}$} & $48.04 \pm 9.90$ & $47.03 \pm 9.23$ & $45.59 \pm 8.50$ & $\mathrm{Val} / \mathrm{Val}>\mathrm{Val} / \mathrm{Met}$ & 0.04 \\
\hline & & & & $\mathrm{Val} / \mathrm{Val}>\mathrm{Met} /$ Met & \\
\hline V & $50.70 \pm 10.95$ & $53.76 \pm 11.12$ & $51.36 \pm 10.72$ & $\mathrm{Val} / \mathrm{Val}<\mathrm{Val} / \mathrm{Met}$ & 0.00 \\
\hline \multirow[t]{2}{*}{$\mathrm{F}$} & $50.67 \pm 10.89$ & $48.21 \pm 9.85$ & $47.34 \pm 8.09$ & $\mathrm{Val} / \mathrm{Val}>\mathrm{Val} / \mathrm{Met}$ & 0.00 \\
\hline & & & & $\mathrm{Val} / \mathrm{Val}>\mathrm{Met} /$ Met & 0.00 \\
\hline C & $48.09 \pm 10.15$ & $47.64 \pm 10.69$ & $47.93 \pm 9.22$ & & \\
\hline \multicolumn{6}{|c|}{ POMS (electrical stimulation) } \\
\hline T-A & $48.33 \pm 11.22$ & $47.45 \pm 8.74$ & $47.06 \pm 9.01$ & & \\
\hline $\mathrm{D}$ & $50.18 \pm 10.89$ & $48.79 \pm 9.39$ & $48.14 \pm 8.83$ & $\mathrm{Val} / \mathrm{Val}>\mathrm{Met} / \mathrm{Met}$ & 0.01 \\
\hline \multirow[t]{2}{*}{$\mathrm{A}-\mathrm{H}$} & $48.02 \pm 10.01$ & $47.09 \pm 9.24$ & $45.37 \pm 8.40$ & $\mathrm{Val} / \mathrm{Val}>\mathrm{Val} / \mathrm{Met}$ & 0.01 \\
\hline & & & & $\mathrm{Val} / \mathrm{Val}>\mathrm{Met} / \mathrm{Met}$ & 0.00 \\
\hline \multirow[t]{2}{*}{$\mathrm{V}$} & $50.36 \pm 10.83$ & $53.74 \pm 11.13$ & $51.55 \pm 10.88$ & $\mathrm{Val} / \mathrm{Val}<\mathrm{Val} / \mathrm{Met}$ & 0.00 \\
\hline & & & & $\mathrm{Val} /$ Met $>$ Met/Met & 0.00 \\
\hline \multirow[t]{2}{*}{$\mathrm{F}$} & $50.76 \pm 11.00$ & $48.25 \pm 9.84$ & $47.28 \pm 8.13$ & $\mathrm{Val} / \mathrm{Val}>\mathrm{Val} / \mathrm{Met}$ & 0.00 \\
\hline & & & & $\mathrm{Val} / \mathrm{Val}>\mathrm{Met} / \mathrm{Met}$ & 0.00 \\
\hline C & $48.31 \pm 10.15$ & $47.79 \pm 10.70$ & $47.99 \pm 9.27$ & & \\
\hline
\end{tabular}

Note: Data represent mean + /- standard deviation unless otherwise noted.

Abbreviations: BDNF, brain-derived neurotrophic factor; POMS, Profile of Mood States; TSST, Trier Social Stress Test; T-A, tension-anxiety; D, depression-dejection; A-H, anger-hostility; $\mathrm{V}$, vigor; $\mathrm{F}$, fatigue; $\mathrm{C}$, confusion.

Beck Depression Inventory scores $(F=0.11, P=0.89, d f=1)$, STAI-T score before TSST ( $F=1.23, P=0.33, d f=1)$ STAI-T score before electrical stimulation $(F=1.03, P=0.36, d f=1)$, STAI-S scores before TSST $(F=1.79, P=0.17, d f=1)$, and STAI-S scores before electrical stimulation $(F=1.80, P=0.17$, $d f=1$ ) (Table 1).

With respect to the POMS test, levels of tension-anxiety and fatigue before TSST in the Val/Val group were significantly higher than those in the Val/Met and Met/Met groups (Table 2). Levels of vigor before TSST in the Val/Val were significantly lower than those in the Val/Met group. Levels of depression-dejection before electrical stimulation in the $\mathrm{Val} / \mathrm{Val}$ group were significantly higher than those in the $\mathrm{Val} / \mathrm{Met}$ and Met/Met groups. Levels of tension-anxiety and fatigue before electrical stimulation in the Val/Val group were significantly higher than those in Val/Met and Met/Met groups. Levels of vigor before electrical stimulation in the $\mathrm{Val} / \mathrm{Val}$ and Met/Met groups were significantly lower than those in the Val/Met group.

There were no significant differences in NEO-PI-R and TCI among the Val/Val, Val/Met, and Met/Met groups (Table 3).

Table 3 NEO-PI-R and TCl scores for the three BDNF genotype groups

\begin{tabular}{|c|c|c|c|c|c|}
\hline Characteristic & $\mathrm{Val} / \mathrm{Val}(\mathrm{n}=46)$ & Val/Met $(n=85)$ & Met/Met $(n=97)$ & $\boldsymbol{F}$ & $P$ \\
\hline \multicolumn{6}{|l|}{ NEO-PI-R } \\
\hline Neuroticism & $53.26 \pm 10.92$ & $53.91 \pm 11.48$ & $53.62 \pm 12.17$ & 0.08 & 0.92 \\
\hline Extraversion & $46.06 \pm I I .58$ & $44.85 \pm 11.97$ & $43.24 \pm|2.3|$ & 1.57 & 0.21 \\
\hline Openness & $52.62 \pm 8.73$ & $53.34 \pm 8.49$ & $53.02 \pm 9.34$ & 0.18 & 0.84 \\
\hline Agreeableness & $47.95 \pm|0.5|$ & $47.59 \pm 10.59$ & $47.68 \pm 11.57$ & 0.03 & 0.97 \\
\hline Conscientiousness & $39.95 \pm 12.17$ & $40.60 \pm 12.42$ & $39.45 \pm 10.38$ & 0.38 & 0.69 \\
\hline \multicolumn{6}{|l|}{$\mathrm{TCl}$} \\
\hline Novelty seeking & $53.26 \pm 10.92$ & $53.91 \pm 11.48$ & $53.62 \pm 12.17$ & 0.54 & 0.58 \\
\hline Harm avoidance & $46.06 \pm 11.58$ & $44.85 \pm 11.97$ & $43.24 \pm|2.3|$ & 0.45 & 0.64 \\
\hline Reward dependence & $52.62 \pm 8.73$ & $53.34 \pm 8.49$ & $53.02 \pm 9.34$ & 0.06 & 0.94 \\
\hline Persistence & $47.95 \pm|0.5|$ & $47.59 \pm 10.59$ & $47.68 \pm I I .57$ & 1.34 & 0.26 \\
\hline Self-directedness & $39.95 \pm 12.17$ & $40.60 \pm 12.42$ & $39.45 \pm 10.38$ & 0.14 & 0.87 \\
\hline Cooperativeness & $53.26 \pm 10.92$ & $53.91 \pm 11.48$ & $53.62 \pm 12.17$ & 0.01 & 0.99 \\
\hline Self-transcendence & $46.06 \pm I 1.58$ & $44.85 \pm 11.97$ & $43.24 \pm|2.3|$ & 0.05 & 0.95 \\
\hline
\end{tabular}

Note: Data represent mean \pm standard deviation unless otherwise noted.

Abbreviations: BDNF, brain-derived neurotrophic factor; NEO-PI-R, Neuroticism, Extraversion, and Openness Personality Inventory Revised; TCI, Temperament and Character Inventory. 
Table 4 Effects of BDNF genotype on heart rate variability

\begin{tabular}{|c|c|c|c|c|c|c|}
\hline Test & $\mathrm{Val} / \mathrm{Val}(\mathrm{n}=\mathbf{4 7})$ & Val/Met $(n=108)$ & Met/Met $(n=56)$ & $\boldsymbol{F}$ & $\mathbf{P}$ & Post hoc \\
\hline \multicolumn{7}{|l|}{$\overline{T S S T}$} \\
\hline Low-frequency power, $\mathrm{ms}^{2} / \mathrm{Hz}$ & $60.10 \pm 16.83$ & $59.18 \pm 17.00$ & $51.44 \pm 17.91$ & 4.28 & 0.02 & $\mathrm{Val} / \mathrm{Val}>\mathrm{Val} / \mathrm{Met} \sim \mathrm{Met} / \mathrm{Met}$ \\
\hline High-frequency power, $\mathrm{ms}^{2} / \mathrm{Hz}$ & $48.56 \pm 17.91$ & $40.82 \pm 17.00$ & $39.90 \pm 16.83$ & 4.52 & 0.01 & $\mathrm{Val} / \mathrm{Val}>\mathrm{Val} / \mathrm{Met} \sim \mathrm{Met} / \mathrm{Met}$ \\
\hline Low-frequency ratio, $\mathrm{ms}^{2} / \mathrm{Hz}$ & $1.41 \pm 1.05$ & $2.04 \pm 1.80$ & $2.22 \pm 2.26$ & 3.22 & 0.04 & $\mathrm{Val} / \mathrm{Val}<\mathrm{Met} / \mathrm{Met}$ \\
\hline \multicolumn{7}{|l|}{ Electrical stimulation } \\
\hline Low-frequency power, $\mathrm{ms}^{2} / \mathrm{Hz}$ & $50.94 \pm|7.9|$ & $52.04 \pm 19.10$ & $51.78 \pm 18.64$ & 0.01 & 0.91 & \\
\hline High-frequency power, $\mathrm{ms}^{2} / \mathrm{Hz}$ & $49.06 \pm|7.9|$ & $47.96 \pm 19.10$ & $47.99 \pm 18.97$ & 0.35 & 0.90 & \\
\hline Low-frequency ratio, $\mathrm{ms}^{2} / \mathrm{Hz}$ & $1.47 \pm 1.38$ & $1.81 \pm 3.34$ & $1.67 \pm 2.09$ & 0.49 & 0.61 & \\
\hline
\end{tabular}

Abbreviations: BDNF, brain-derived neurotrophic factor; TSST, Trier Social Stress Test.

\section{Heart rate variability}

LF and HF power in the Val/Met and Met/Met groups were significantly less than in the $\mathrm{Val} / \mathrm{Val}$ group in the TSST $(F=4.28, P=0.02, d f=1$; and $F=4.52, P=0.01, d f=1$, respectively; Table 4). LF/HF in the Met/Met group was significantly greater than that in $\mathrm{Val} / \mathrm{Val}$ group in the TSST $(F=3.22, P=0.04, d f=1)$. There were no differences in the power of $\mathrm{LF}$ or $\mathrm{HF}$ or in the $\mathrm{LF} / \mathrm{HF}$ ratio among the $\mathrm{Val} / \mathrm{Val}$, $\mathrm{Val} / \mathrm{Met}$, and Met/Met groups in the electrical stimulation stress test $(F=0.01, P=0.91, d f=1 ; F=0.35, P=0.90, d f=1$; and $F=0.49, P=0.61, d f=1$, respectively).

\section{TSST}

No differences were observed in the sAA responses between women and men among the Val/Val, Val/Met, and Met/ Met groups in the TSST $\left(F_{(2,263)}=0.37, P=0.69\right.$ [Figure 1A]; $F_{(2,410)}=0.10, P=0.91$ [Figure 1B], respectively). Women in the Met/Met group had a stronger trend of salivary cortisol responses than those in the $\mathrm{Val} / \mathrm{Met}$ and $\mathrm{Val} / \mathrm{Val}$ groups in the TSST $\left(F_{(2,263)}=2.39, P=0.09\right.$ [Figure 2A] $)$ and men in the Met/Met group had significantly stronger salivary cortisol

A

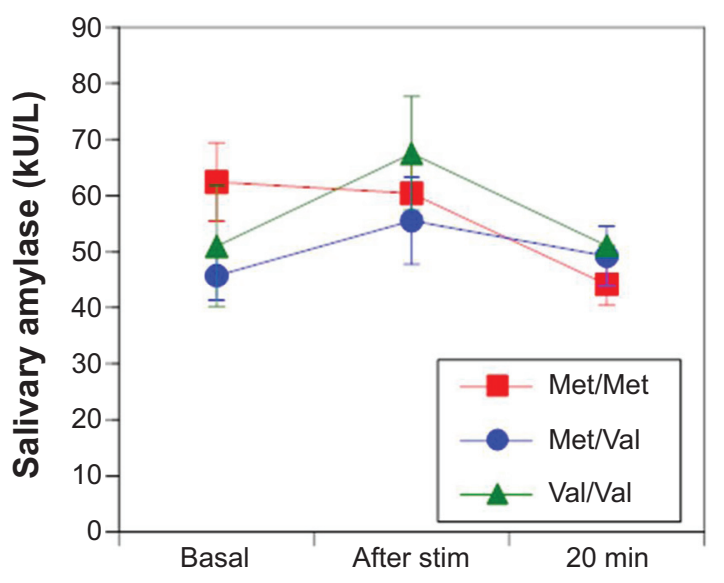

responses than those in the $\mathrm{Val} / \mathrm{Met}$ and $\mathrm{Val} / \mathrm{Val}$ groups $\left(F_{(2,413)}=3.19, P=0.04\right.$ [Figure 2B] $)$.

\section{Electrical stimulation}

No differences were observed in sAA responses in women $\left(F_{(2,494)}=1.62 ; P=0.20\right.$ [Figure 3A] $)$ and men $\left(F_{(2,701)}=0.13\right.$; $P=0.88$ [Figure 3B]) among the $\mathrm{Val} / \mathrm{Val}, \mathrm{Val} / \mathrm{Met}$, and Met/Met groups during electrical stimulation. Likewise, no differences were observed in salivary cortisol responses between women $\left(F_{(2377)}=0.61 ; P=0.54\right.$ [Figure 4A] $)$ and men $\left(F_{(2,584)}=0.03 ; P=0.97\right.$ [Figure 4B]) among the $\mathrm{Val} / \mathrm{Val}, \mathrm{Val} /$ Met, and Met/Met groups after electrical stimulation.

\section{Discussion}

Our results agree with the findings of a small number of studies that indicate that the HPA axis response to laboratory-induced social stress is modulated by common polymorphisms..$^{50,51,62}$ In addition, we report that the BDNF Val66Met polymorphism has almost the same effects on salivary cortisol in women and men. The women and men in the Met/Met group showed significantly stronger salivary

B

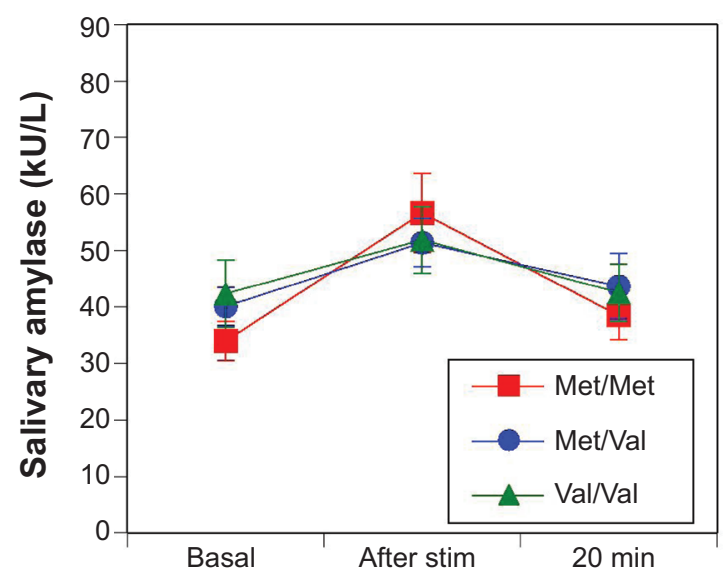

Figure I In the Trier Social Stress Test, there were no differences in female salivary $\alpha$-amylase responses among the $\mathrm{Val} / \mathrm{Val}$, $\mathrm{Val} / \mathrm{Met}$, and $\mathrm{Met} / \mathrm{Met}$ groups $\left(F_{(2,263)}=0.37\right.$;

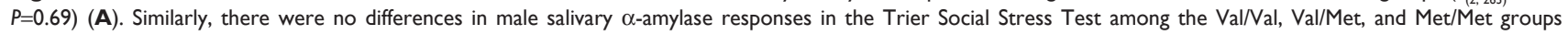
$\left(F_{(2,410)}=0.10 ; P=0.91\right)(\mathbf{B})$.

Abbreviations: min, minute; stim, stimulation. 
A

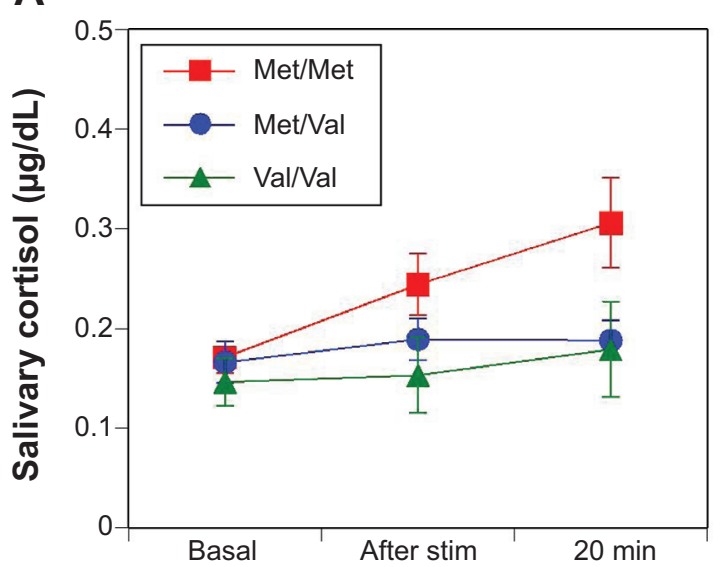

B

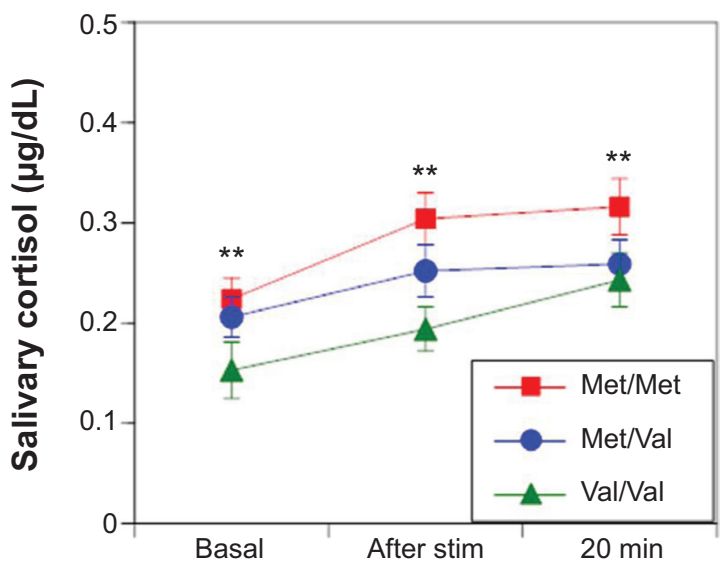

Figure 2 In the Trier Social Stress Test, female Met/Met individuals have a stronger salivary cortisol response than $\mathrm{Val} / \mathrm{Met}$ and $\mathrm{Val} / \mathrm{Val}$ individuals $\left(F_{(2.263)}=2.39 ; P=0.09\right)$ (A). Male Met/Met individuals showed stronger salivary cortisol responses than $\mathrm{Val} / \mathrm{Met}$ and $\mathrm{Val} / \mathrm{Val}$ individuals in the Trier Social $S$ tress $\mathrm{Test}\left(F_{(2,413)}=3.19 ; \mathrm{P}=0.04\right)(\mathbf{B})$. $* * P<0.01$.

Abbreviations: min, minute; stim, stimulation.

cortisol responses in the TSST than those in the Val/Met and $\mathrm{Val} / \mathrm{Val}$ groups.

Bryant et al reported that increased salivary cortisol response after the stressor challenge suggested intrusive memories of emotional stimuli in men, but not in women. ${ }^{63}$ Shalev et al reported a stronger cortisol response to TSST in men than women. ${ }^{64}$ In men, Val/Val homozygotes showed a larger increase in salivary cortisol than Val/Met heterozygotes. In women, a tendency toward the lowest rise for the contrary response was observed in the Val/Val homozygotes. Alexander et al reported that carriers of the Met allele showed a significantly attenuated HPA axis and cardiovascular reactivity to psychosocial stressors compared with individuals with the Val/Val genotype. ${ }^{65}$ In other words, the weak cortisol response in women tended to obscure the differences as a result of the allele, and the stronger cortisol response in men revealed the differences of the alleles.

This is a contradictory outcome because sex-based differences in psychiatry point to the higher incidence of mood and anxiety disorders in women. However, numerous epidemiological studies show that the number of men is about twice that of women for individual disorders. ${ }^{66}$ There is an exact sex $\times$ genotype effect interaction between BDNF Val66Met polymorphism and environmental stress on depression. ${ }^{67,68}$ Shalev et $\mathrm{al}^{64}$ reported there is a conflict between high anxiety and low cortisol response in the TSST in men and between the low and high cortisol response in the TSST anxiety in women. Analysis of genetic data might explain that sex-based differences induce stress-related cortisol responses. These workers reported that salivary cortisol showed a greater
A

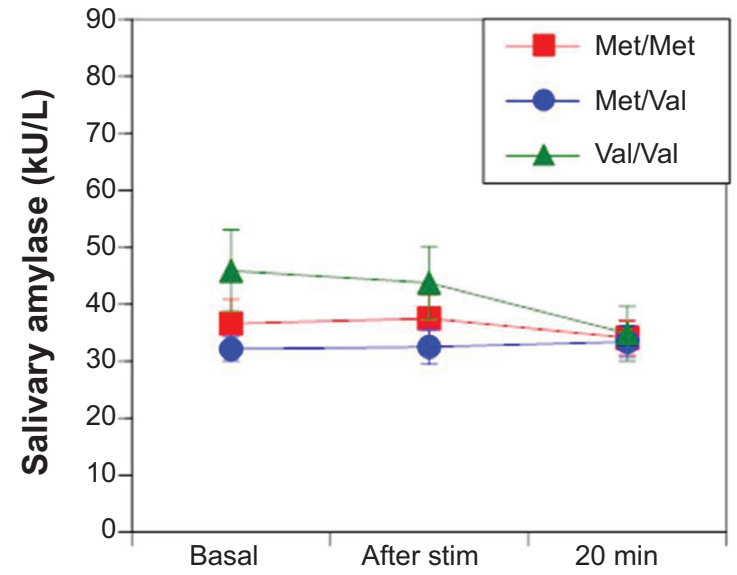

B

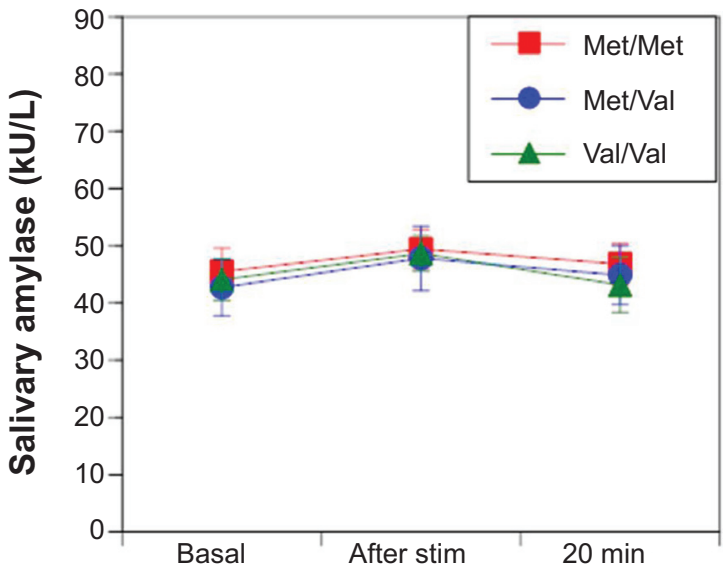

Figure 3 In the electrical stimulation stress test, there were no differences in female salivary $\alpha$-amylase responses among the $\mathrm{Val} / \mathrm{Val}$, $\mathrm{Val} / \mathrm{Met}$, and $\mathrm{Met} / \mathrm{Met}$ groups $\left(F_{(2,494)}=\mathrm{I} .62\right.$; $P=0.20)(\mathbf{A})$. Similarly, there were no differences in male salivary $\alpha$-amylase responses among the $\mathrm{Val} / \mathrm{Val}$, $\mathrm{Val} / \mathrm{Met}$, and $\mathrm{Met} / \mathrm{Met} \mathrm{groups}\left(F_{(2,701)}=0.13 ; P=0.88\right)(\mathbf{B})$. Abbreviations: min, minute; stim, stimulation. 
A

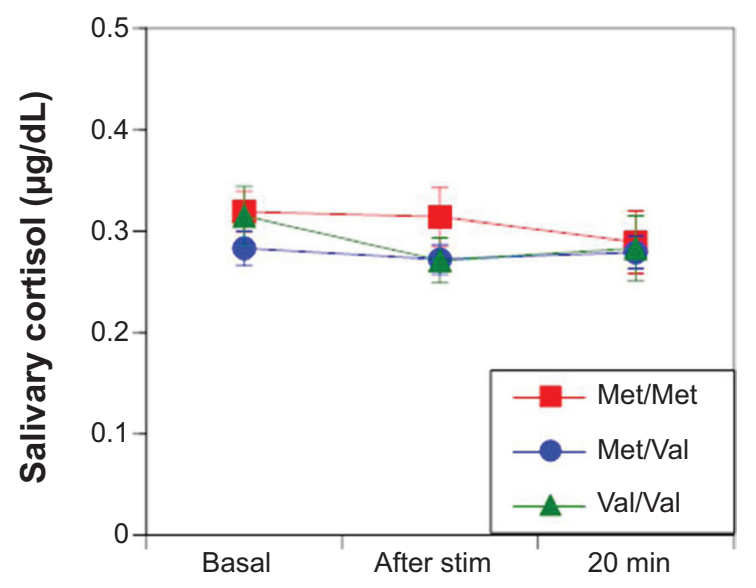

B

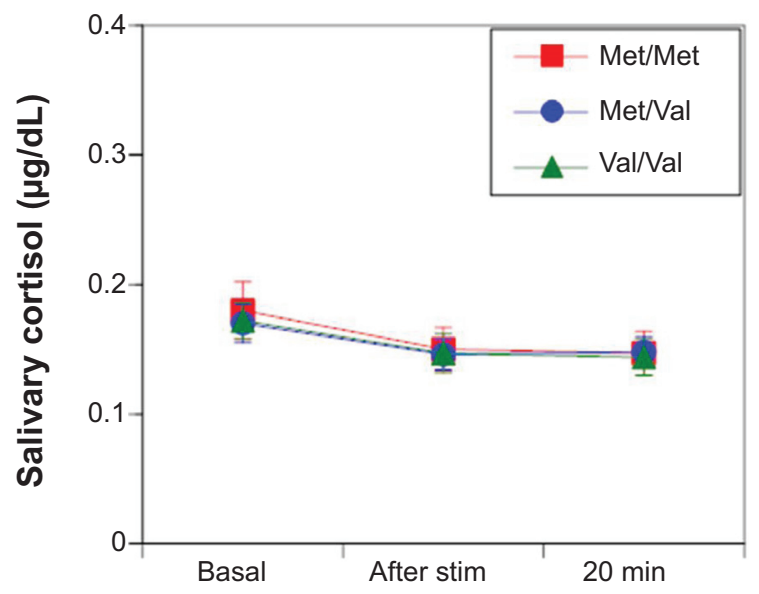

Figure 4 After the electrical stimulation stress test, there were no differences in female salivary cortisol responses among the $\mathrm{Val} / \mathrm{Val}$, Val/Met, and Met/Met groups $\left(F_{(2,377)}=0.61 ; P=0.54\right)(\mathbf{A})$. Similarly, there were no differences in male salivary cortisol responses among the $\mathrm{Val} / \mathrm{Val}$, $\mathrm{Val} / \mathrm{Met}$, and Met/Met groups $\left(F_{(2,584)}=0.03 ; P=0.97\right)(\mathbf{B})$.

response in TSST in men than in women. The salivary cortisol response in the TSST was greater in BDNF Val/Val men than in BDNF Val/Met men. Conversely, the salivary cortisol response in the TSST in BDNF Val/Val women was lower than in BDNF Val/Met women. The results of this study do not agree with our results: salivary cortisol response was higher than in BDNF Met/Met than in Met/Val or Val/Val individuals. The two studies used almost the same methods (sample size, age, TSST protocol, and salivary amylase analysis). The ethnic variation in participants may have led to different results. These studies suggest genetics may lead to contradictory responses regarding sex-based differences in psychological stress responses.

The BDNF Val66Met polymorphism alters the intracellular packaging of proBDNF and reduces the activity-dependent secretion of mature BDNF in primary cortical neurons. ${ }^{6}$ Some studies on psychiatric disorders reported the Met allele was associated with reduced BDNF levels, ${ }^{70,71}$ whereas other studies in healthy individuals showed increased BDNF levels in Met-allele carriers. ${ }^{72,73}$ These studies suggest the association between the Val/Met polymorphism and the BDNF secretion may differ between patients and controls.

We demonstrated here that there are no significant differences in STAI traits among Val/Val, Val/Met, and Met/Met subjects before TSST and electrical stimulation. This result is consistent with reports that BDNF Val66Met showed no significant association with STAI-T scores. ${ }^{74}$ In contrast, Lang et $\mathrm{al}^{37}$ observed that genotype had a significant effect of the phenotype, with higher levels of trait anxiety in $\mathrm{Val} / \mathrm{Val}$ compared with Val/Met and Met/Met subjects, as observed in the trait-related anxiety score in STAI. ${ }^{33}$ Trait anxiety in the STAI may not only develop into pure anxiety but also include factors that reveal depression and other negative consequences. ${ }^{75}$ Our results do not show differences in the Beck Depression Inventory scores among the $\mathrm{Val} / \mathrm{Val}$, Val/ Met, and Met/Met genotypes. Another study on undergraduate university students from various fields reported that the BDNF Val66Met polymorphism showed no significant association with STAI-T scores, using undergraduate students from all fields of study at a university. ${ }^{76}$

Our results show that the levels of depression-dejection in POMS before electrical stimulation were significantly higher in Val/Val individuals than Met/Met individuals. Levels of vigor in POMS before TSST or electrical stimulation were significantly lower in $\mathrm{Val} / \mathrm{Val}$ individuals than in Met-allele carriers. We also show that the levels of tension-anxiety and fatigue in POMS before TSST or electrical stimulation are significantly higher in $\mathrm{Val} / \mathrm{Val}$ individuals than in Met carriers. These results suggest that individuals with the $\mathrm{Val} / \mathrm{Val}$ genotype have more anxiety and fatigue, and less vigor.

Our data show there are no significant differences in neuroticism, extraversion, openness, agreeableness, and conscientiousness of NEO-PI-R among the Val/Val, $\mathrm{Val} / \mathrm{Met}$, and Met/Met genotypes before TSST and electrical stimulation. These results are consistent with the report by Sen et al, ${ }^{, 7}$ although the association between the BDNF Val66Met polymorphism and neuroticism that was demonstrated in that study was not replicated in the present study. Aside from a study showing an association between BDNF Val66Met and neuroticism, no major studies reported such an association, ${ }^{37}$ although Itoh et al reported a significant association between extraversion and the BDNF Val66Met genotype ${ }^{81}$ However, 
the present results are consistent with the findings of earlier studies, ${ }^{76,78,79}$ with the exception of a study by Terracciano et al. ${ }^{78}$ Recently, De Beaumont et al reported that carriers of the BDNF Met allele were significantly less extraverted (and thus more introverted) than BDNF Val homozygotes. ${ }^{79}$ Further research is required with larger sample sizes to resolve these discrepancies.

We demonstrated here that there are no significant differences in novelty seeking, harm avoidance, reward dependence, persistence, self-directedness, cooperativeness, and self-transcendence of TCI among the Val/Val, Val/ Met, and Met/Met genotypes before TSST and electrical stimulation. Tochigi et al reported that the BDNF Val66Met polymorphism might be associated with personality traits of reward dependence of TCI in female, but not male, healthy individuals. ${ }^{80}$ In contrast, other authors reported no significant differences in TCI scores among Val/Val, Val/Met, and Met/ Met genotypes. ${ }^{81-83}$ In addition, Minelli et al reported that a significant correlation exists between high scores of harm avoidance of TCI and low serum BDNF levels. ${ }^{84}$ Further studies with larger sample sizes are required to determine the influence of the BDNF Val66Met polymorphism and BDNF protein levels on the psychological responses to stressful life events.

Our results show that in TSST, the HF power of heart rate variability is significantly less in Met/Met than in Val/ Val individuals, whereas the ratio of LF/HF power is significantly greater in Met/Met than in Val/Val individuals. Yang et al reported that decreased HF power and increased LF/HF ratio are observed in individuals with the Met/Met genotype compared with individuals with the Val/Val genotype. ${ }^{85}$ These findings suggest an altered sympathovagal balance with reduced parasympathetic modulation and possibly increased sympathetic activity. This suggests the requirement of further investigations into the relationship between the BDNF-associated autonomic nervous system imbalance and the incidence of anxiety disorders and cardiovascular diseases.

Our study has some limitations. First, the number of healthy control subjects was relatively small; we are planning a larger study. Second, the number of hormonal assessments was limited, and therefore we must increase the number of hormonal responses tested. Third, we did not examine the serum BDNF levels. Fourth, we set the period before occurrence of the stress as the baseline; sAA and salivary cortisol levels may have been influenced by the length of stay in the hospital. Fifth, although the BDNF Val66Met polymorphism has been reported to impair intracellular trafficking and activity-dependent secretion of BDNF, ${ }^{69}$ the difference in the serum BDNF levels among the Val/Val, Val/Met, and Met/ Met groups is controversial. Importantly, whether the serum BDNF levels in response to psychological stress, such as TSST and the electrical stress test, are affected by the BDNF Val66Met polymorphism is unknown. Therefore, serum BDNF levels should be measured before and after the stress test in $\mathrm{Val} / \mathrm{Val}$, Val/Met, and Met/Met groups to determine their association with BDNF Val66Met polymorphism.

In conclusion, these preliminary results show an association between genes, hormonal responses, and personality and suggest that in the TSST, Met/Met individuals have a significantly stronger salivary cortisol response, but not sAA response, compared with $\mathrm{Val} / \mathrm{Met}$ and $\mathrm{Val} / \mathrm{Val}$ individuals. Thus, the relationship between salivary cortisol and stress may be affected by the significant biological variant of BDNF, the G196A SNP (Val66Met).

\section{Acknowledgments}

The authors thank Ms Kazumi Oda for invaluable technical assistance with the assays for sAA and salivary cortisol. The study was supported by a grant from the Grant-in-Aid for Scientific Research (23591719) of the Ministry of Health and Welfare, Japan.

\section{Disclosure}

The authors declare no conflicts of interest in this work.

\section{References}

1. Herman JP, Cullinan WE. Neurocircuitry of stress: central control of the hypothalamo-pituitary-adrenocortical axis. Trends Neurosci. 1997; 20(2):78-84.

2. Isogawa $\mathrm{K}$, Tsuru J, Tanaka $\mathrm{Y}$, et al. Association between salivary amylase, cortisol and stress. In: Davies RS, editor. Handbook of Neuropsychiatry Research. Hauppauge, NY: Nova Science Publishers; 2010: 113-123.

3. Tasker JG, Herman JP. Mechanisms of rapid glucocorticoid feedback inhibition of the hypothalamic-pituitary-adrenal axis. Stress. 2011; 14(4):398-406.

4. Kirschbaum C, Hellhammer DH. Salivary cortisol in psychoneuroendocrine research: recent developments and applications. Psychoneuroendocrinology. 1994;19(4):313-333.

5. Stahl F, Dörner G. Responses of salivary cortisol levels to stresssituations. Endokrinologie. 1982;80(2):158-162.

6. Perroni F, Tessitore A, Cibelli G, et al. Effects of simulated firefighting on the responses of salivary cortisol, alpha-amylase and psychological variables. Ergonomics. 2009;52(4):484-491.

7. van Stegeren AH, Wolf OT, Kindt M. Salivary alpha amylase and cortisol responses to different stress tasks: impact of sex. Int J Psychophysiol. 2008;69(1):33-40.

8. van Stegeren A, Rohleder N, Everaerd W, Wolf OT. Salivary alpha amylase as marker for adrenergic activity during stress: effect of betablockade. Psychoneuroendocrinology. 2006;31(1):137-141.

9. Granger DA, Kivlighan KT, el-Sheikh M, Gordis EB, Stroud LR. Salivary alpha-amylase in biobehavioral research: recent developments and applications. Ann N Y Acad Sci. 2007;1098(1):122-144. 
10. Schumacher J, Jamra RA, Becker T, et al. Evidence for a relationship between genetic variants at the brain-derived neurotrophic factor (BDNF) locus and major depression. Biol Psychiatry. 2005;58(4):307-314.

11. Chatterton RT Jr, Vogelsong KM, Lu YC, Hudgens GA. Hormonal responses to psychological stress in men preparing for skydiving. $J$ Clin Endocrinol Metab. 1997;82(8):2503-2509.

12. Skosnik PD, Chatterton RT Jr, Swisher T, Park S. Modulation of attentional inhibition by norepinephrine and cortisol after psychological stress. Int J Psychophysiol. 2000;36(1):59-68.

13. Bosch JA, Brand HS, Ligtenberg TJ, Bermond B, Hoogstraten J, Nieuw Amerongen AV. Psychological stress as a determinant of protein levels and salivary-induced aggregation of Streptococcus gordonii in human whole saliva. Psychosom Med. 1996;58(4):374-382.

14. BoschJA, de Geus EJ, Veerman EC, Hoogstraten J, Nieuw Amerongen AV. Innate secretory immunity in response to laboratory stressors that evoke distinct patterns of cardiac autonomic activity. Psychosom Med. 2003;65(2):245-258.

15. Rohleder N, Nater UM, Wolf JM, Ehlert U, Kirschbaum C. Psychosocial stress-induced activation of salivary alpha-amylase: an indicator of sympathetic activity? Ann N Y Acad Sci. 2004;1032(1):258-263.

16. Nater UM, Rohleder N, Gaab J, et al. Human salivary alpha-amylase reactivity in a psychosocial stress paradigm. Int J Psychophysiol. 2005;55(3):333-342.

17. Nater UM, La Marca R, Florin L, et al. Stress-induced changes in human salivary alpha-amylase activity - associations with adrenergic activity. Psychoneuroendocrinology. 2006;31(1):49-58.

18. Takai N, Yamaguchi M, Aragaki T, Eto K, Uchihashi K, Nishikawa Y. Effect of psychological stress on the salivary cortisol and amylase levels in healthy young adults. Arch Oral Biol. 2004;49(12):963-968.

19. Ehlert U, Erni K, Hebisch G, Nater U. Salivary alpha-amylase levels after yohimbine challenge in healthy men. J Clin Endocrinol Metab. 2006;91(12):5130-5133.

20. Engert V, Vogel S, Efanov SI, et al. Investigation into the cross-correlation of salivary cortisol and alpha-amylase responses to psychological stress. Psychoneuroendocrinology. 2011;36(9):1294-1302.

21. Sapolsky RM, Romero LM, Munck AU. How do glucocorticoids influence stress responses? Integrating permissive, suppressive, stimulatory, and preparative actions. Endocr Rev. 2000;21(1):55-89.

22. Takai N, Yamaguchi M, Aragaki T, Eto K, Uchihashi K, Nishikawa Y. Gender-specific differences in salivary biomarker responses to acute psychological stress. Ann N Y Acad Sci. 2007;1098(1):510-515.

23. Kumsta R, Entringer S, Koper JW, van Rossum EF, Hellhammer DH, Wüst S. Sex specific associations between common glucocorticoid receptor gene variants and hypothalamus-pituitary-adrenal axis responses to psychosocial stress. Biol Psychiatry. 2007;62(8):863-869.

24. Uhart M, McCaul ME, Oswald LM, Choi L, Wand GS. GABRA6 gene polymorphism and an attenuated stress response. Mol Psychiatry. 2004;9(11):998-1006.

25. Drummond PD. Involvement of the sympathetic nervous system in complex regional pain syndrome. Int J Low Extrem Wounds. 2004;3(1): $35-42$.

26. Desborough JP. The stress response to trauma and surgery. Br J Anaesth. 2000;85(1):109-117.

27. Roy RA, Boucher JP, Comtois AS. Heart rate variability modulation after manipulation in pain-free patients vs patients in pain. JManipulative Physiol Ther. 2009;32(4):277-286.

28. Shirasaki S, Fujii H, Takahashi M, et al. Correlation between salivary alpha-amylase activity and pain scale in patients with chronic pain. Reg Anesth Pain Med. 2007;32(2):120-123.

29. Tanaka Y, Ishitobi Y, Maruyama Y, et al. Salivary alpha-amylase and cortisol responsiveness following electrical stimulation stress in major depressive disorder patients. Prog Neuropsychopharmacol Biol Psychiatry. 2012a;36(2):220-224.

30. Campbell J, Ehlert U. Acute psychosocial stress: does the emotional stress response correspond with physiological responses? Psychoneuroendocrinology. 2012;37(8):1111-1134.

31. Dechant G, Neumann H. Neurotrophins. Adv Exp Med Biol. 2002;513: 303-334.
32. Lipsky RH, Marini AM. Brain-derived neurotrophic factor in neuronal survival and behavior-related plasticity. Ann N Y Acad Sci. 2007;1122(1): 130-143.

33. Bergström A, Jayatissa MN, Mørk A, Wiborg O. Stress sensitivity and resilience in the chronic mild stress rat model of depression; an in situ hybridization study. Brain Res. 2008;1196:41-52.

34. Seidah NG, Benjannet S, Pareek S, Chrétien M, Murphy RA. Cellular processing of the neurotrophin precursors of NT3 and BDNF by the mammalian proprotein convertases. FEBS Lett. 1996;379(3):247-250.

35. Egan MF, Kojima M, Callicott JH, et al. The BDNF val66met polymorphism affects activity-dependent secretion of BDNF and human memory and hippocampal function. Cell. 2003;112(2):257-269.

36. Bueller JA, Aftab M, Sen S, Gomez-Hassan D, Burmeister M, Zubieta JK. BDNF Val66Met allele is associated with reduced hippocampal volume in healthy subjects. Biol Psychiatry. 2006;59(9):812-815.

37. Lang UE, Hellweg R, Kalus P, et al. Association of a functional BDNF polymorphism and anxiety-related personality traits. Psychopharmacology (Berl). 2005;180(1):95-99.

38. Monteggia LM, Luikart B, Barrot M, et al. Brain-derived neurotrophic factor conditional knockouts show gender differences in depressionrelated behaviors. Biol Psychiatry. 2007;61(2):187-197.

39. Frodl T, Schüle C, Schmitt G, et al. Association of the brain-derived neurotrophic factor Val66Met polymorphism with reduced hippocampal volumes in major depression. Arch Gen Psychiatry. 2007;64(4):410-416.

40. Sarchiapone M, Carli V, Roy A, et al. Association of polymorphism (Val66Met) of brain-derived neurotrophic factor with suicide attempts in depressed patients. Neuropsychobiology. 2008;57(3):139-145.

41. Gatt JM, Nemeroff CB, Dobson-Stone C, et al. Interactions between BDNF Val66Met polymorphism and early life stress predict brain and arousal pathways to syndromal depression and anxiety. Mol Psychiatry. 2009;14(7):681-695.

42. Choi MJ, Kang RH, Lim SW, Oh KS, Lee MS. Brain-derived neurotrophic factor gene polymorphism (Val66Met) and citalopram response in major depressive disorder. Brain Res. 2006;1118(1):176-182.

43. Tsai SJ, Hong CJ, Liou YJ. Effects of BDNF polymorphisms on antidepressant action. Psychiatry Investig. 2010;7(4):236-242.

44. Groves JO. Is it time to reassess the BDNF hypothesis of depression? Mol Psychiatry. 2007;12(12):1079-1088.

45. Bradley MM, Codispoti M, Sabatinelli D, Lang PJ. Emotion and motivation II: sex differences in picture processing. Emotion. 2001; 1(3):300-319.

46. Cahill L, van Stegeren A. Sex-related impairment of memory for emotional events with beta-adrenergic blockade. Neurobiol Learn Mem. 2003; 79(1):81-88.

47. Canli T, Desmond JE, Zhao Z, Gabrieli JD. Sex differences in the neural basis of emotional memories. Proc Natl Acad Sci U S A. 2002; 99(16):10789-10794.

48. van Stegeren AH, Everaerd W, Cahill L, McGaugh JL, Gooren LJ. Memory for emotional events: differential effects of centrally versus peripherally acting beta-blocking agents. Psychopharmacology (Berl). 1998;138(3-4):305-310.

49. Kudielka BM, Kirschbaum C. Sex differences in HPA axis responses to stress: a review. Biol Psychol. 2005;69(1):113-132.

50. Kivlighan KT, Granger DA. Salivary alpha-amylase response to competition: relation to gender, previous experience, and attitudes. Psychoneuroendocrinology. 2006;31(6):703-714.

51. Chong RY, Oswald L, Yang X, Uhart M, Lin PI, Wand GS. The mu-opioid receptor polymorphism A118G predicts cortisol responses to naloxone and stress. Neuropsychopharmacology. 2006;31(1):204-211.

52. Tanaka Y, Ishitobi Y, Maruyama Y, et al. Salivary $\alpha$-amylase and cortisol responsiveness following electrical stimulation stress in panic disorder patients. Neurosci Res. 2012b;73(1):80-84.

53. Ishitobi Y, Akiyoshi J, Tanaka Y, et al. Elevated salivary $\alpha$-amylase and cortisol levels in unremitted and remitted depressed patients. Int $J$ Psychiatry Clin Pract. 2010;14(4):268-273. 
54. Hammerfald K, Eberle C, Grau M, et al. Persistent effects of cognitivebehavioral stress management on cortisol responses to acute stress in healthy subjects - a randomized controlled trial. Psychoneuroendocrinology. 2006;31(3):333-339.

55. Inagaki T, Ieda M, Yamashita S, Miyaoka T, Horiguchi J. Salivary alpha-amylase reactivity under psycho-physiological stress. A nonverbal communication measurement tool? J Behavioral Brain Sci. 2011; 1(1):12-15.

56. Yamaguchi M, Kanemori T, Kanemaru M, Takai N, Mizuno Y, Yoshida H. Performance evaluation of salivary amylase activity monitor. Biosens Bioelectron. 2004;20(3):491-497.

57. Yamaguchi M, Deguchi M, Wakasugi J, et al. Hand-held monitor of sympathetic nervous system using salivary amylase activity and its validation by driver fatigue assessment. Biosens Bioelectron. 2006;21(7):1007-1014.

58. Robles TF, Shetty V, Zigler CM, et al. The feasibility of ambulatory biosensor measurement of salivary alpha amylase: Relationships with self-reported and naturalistic psychological stress. Biol Psychol. 2011;86(1):50-56

59. Spielberger C. State-Trait Anxiety Inventory (Form Y). Palo Alto, CA: Mind Garden; 1983.

60. McNair DM, Lorr M, Droppleman LF. EdITS manual for the Profile of Mood States. San Diego: CA: EdITS Educational \& Industrial Testing Service; 1992.

61. Cloninger CR. The temperament and character inventory (TCI): A guide to its development and use. St Louis, MO: Center for Psychobiology of Personality, Washington University; 1994.

62. Gotlib IH, Joormann J, Minor KL, Hallmayer J. HPA axis reactivity: a mechanism underlying the associations among 5-HTTLPR, stress, and depression. Biol Psychiatry. 2008;63(9):847-851.

63. Bryant RA, McGrath C, Felmingham KL. The roles of noradrenergic and glucocorticoid activation in the development of intrusive memories. PLoS ONE. 2013;8(4):e62675.

64. Shalev I, Lerer E, Israel S, et al. BDNF Val66Met polymorphism is associated with HPA axis reactivity to psychological stress characterized by genotype and gender interactions. Psychoneuroendocrinology. 2009;34(3):382-388.

65. Alexander N, Osinsky R, Schmitz A, Mueller E, Kuepper Y, Hennig J. The BDNF Val66Met polymorphism affects HPA-axis reactivity to acute stress. Psychoneuroendocrinology. 2010;35(6):949-953.

66. Valentino RJ, Reyes B, Van Bockstaele E, Bangasser D. Molecular and cellular sex differences at the intersection of stress and arousal. Neuropharmacology. 2012;62(1):13-20.

67. Steel Z, Marnane C, Iranpour C, et al. The global prevalence of common mental disorders: a systematic review and meta-analysis 1980-2013. Int J Epidemiol. 2014;43(2):476-493.

68. Chen J, Li X, McGue M. The interacting effect of the BDNF Val66Met polymorphism and stressful life events on adolescent depression is not an artifact of gene-environment correlation: evidence from a longitudinal twin study. J Child Psychol Psychiatry. 2013;54(10): 1066-1073.

69. Chen ZY, Patel PD, Sant G, et al. Variant brain-derived neurotrophic factor (BDNF) (Met66) alters the intracellular trafficking and activitydependent secretion of wild-type BDNF in neurosecretory cells and cortical neurons. J Neurosci. 2004;24(18):4401-4411.
70. Zhang XY, Chen C, Xiu MH, et al. Cognitive and serum BDNF correlates of BDNF Val66Met gene polymorphism in patients with schizophrenia and normal controls. Hum Genet. 2012;131(7): 1187-1195.

71. Ozan E, Okur H, Eker C, Eker OD, Gönül AS, Akarsu N. The effect of depression, BDNF gene val66met polymorphism and gender on serum BDNF levels. Brain Res Bull. 2010;81(1):61-65.

72. Lang UE, Hellweg R, Sander T, Gallinat J. The Met allele of the BDNF Val66Met polymorphism is associated with increased BDNF serum concentrations. Mol Psychiatry. 2009;14(2):120-122.

73. Bus BA, Arias-Vasquez A, Franke B, Prickaerts J, de Graaf J, Voshaar RC. Increase in serum brain-derived neurotrophic factor in met allele carriers of the BDNF Val66Met polymorphism is specific to males. Neuropsychobiology. 2012;65(4):183-187.

74. Perea CS, Paternina AC, Gomez Y, Lattig MC. Negative affectivity moderated by BDNF and stress response. J Affect Disord. 2012;136(3): 767-774.

75. Bieling PJ, Antony MM, Swinson RP. The State-Trait Anxiety Inventory, Trait version: structure and content re-examined. Behav Res Ther. 1998;36(7-8):777-788.

76. Perea CS, Paternina AC, Gomez Y, Lattig MC. Negative affectivity moderated by BDNF and stress response. J Affect Disord. 2012;136(3): 767-774.

77. Sen S, Nesse RM, Stoltenberg SF, et al. A BDNF coding variant is associated with the NEO personality inventory domain neuroticism, a risk factor for depression. Neuropsychopharmacology. 2003; 28(2):397-401.

78. Terracciano A, Martin B, Ansari D, et al. Plasma BDNF concentration, Val66Met genetic variant and depression-related personality traits. Genes Brain Behav. 2010;9(5):512-518.

79. De Beaumont L, Fiocco AJ, Quesnel G, Lupien S, Poirier J. Altered declarative memory in introverted middle-aged adults carrying the BDNF val66met allele. Behav Brain Res. 2013;253:152-156.

80. Tochigi M, Otowa T, Suga M, et al. No evidence for an association between the BDNF Val66Met polymorphism and schizophrenia or personality traits. Schizophr Res. 2006;87(1-3):45-47.

81. Itoh K, Hashimoto K, Kumakiri C, Shimizu E, Iyo M. Association between brain-derived neurotrophic factor $196 \mathrm{G} / \mathrm{A}$ polymorphism and personality traits in healthy subjects. Am JMed Genet B Neuropsychiatr Genet. 2004;124B(1):61-63.

82. Kim SJ, Cho SJ, Jang HM, et al. Interaction between brain-derived neurotrophic factor Val66Met polymorphism and recent negative stressor in harm avoidance. Neuropsychobiology. 2010;61(1): 19-26.

83. Suzuki A, Matsumoto Y, Shibuya N, et al. The brain-derived neurotrophic factor Val66Met polymorphism modulates the effects of parental rearing on personality traits in healthy subjects. Genes Brain Behav. 2011;10(4):385-391.

84. Minelli A, Zanardini R, Bonvicini C, et al. BDNF serum levels, but not BDNF Val66Met genotype, are correlated with personality traits in healthy subjects. Eur Arch Psychiatry Clin Neurosci. 2011; 261(5):323-329.

85. Yang AC, Chen TJ, Tsai SJ, et al. BDNF Val66Met polymorphism alters sympathovagal balance in healthy subjects. Am J Med Genet B Neuropsychiatr Genet. 2010;153B(5):1024-1030.
Neuropsychiatric Disease and Treatment

\section{Publish your work in this journal}

Neuropsychiatric Disease and Treatment is an international, peerreviewed journal of clinical therapeutics and pharmacology focusing on concise rapid reporting of clinical or pre-clinical studies on a range of neuropsychiatric and neurological disorders. This journa is indexed on PubMed Central, the 'PsycINFO' database and CAS,

\section{Dovepress}

and is the official journal of The International Neuropsychiatric Association (INA). The manuscript management system is completely online and includes a very quick and fair peer-review system, which is all easy to use. Visit http://www.dovepress.com/testimonials.php to read real quotes from published authors. 